\title{
Shaped and multiple beams using planar phased arrays
}

This paper was downloaded from TechRxiv (https://www.techrxiv.org).

\section{LICENSE}

CC BY 4.0

SUBMISSION DATE / POSTED DATE

$12-11-2021 / 17-11-2021$

\section{CITATION}

Sankaravel, Prasad; Meenakshi, M.; Rao, P. Hanumantha (2021): Shaped and multiple beams using planar phased arrays. TechRxiv. Preprint. https://doi.org/10.36227/techrxiv.17001685.v1

$\mathrm{DOI}$ 


\title{
Shaped and multiple beams using planar phased
}

\section{arrays}

\author{
S. Prasad, M. Meenakshi, P. H. Rao,
}

\begin{abstract}
This paper presents a new synthesis and computation method to generate user-specified multiple beams and shaped beams in any arbitrary 3D space. The computation method can generate independently controllable simultaneous multiple beams with arbitrary peak powers. This method is extended to generate arbitrarily shaped beams, using a combination of optimally placed multiple beams at appropriate locations with specific power ratios. An evolutionary algorithm is utilized to optimize the number of beams, peak powers, and their respective positions. This method supports dynamically changeable multiple beams and shaped beam patterns. The proposed method is practically validated on a fully functional integrated $8 \times 8 \mathbf{5 G}$ mmWave tiled phased array in hybrid beamforming architecture to generate scannable single, dual, multiple and shaped beams. Measured results for dual beam with equal and unequal powers at $\left(\theta= \pm 25^{\circ}, \phi=0^{\circ}\right)$ are presented. Measured broad beam with flat pattern over $50^{\circ}$ and $\csc ^{2}$ pattern with $30^{\circ}$ coverage are compared with the proposed computation method results.
\end{abstract}

Index Terms-Shaped beam, multibeam, phased array, beamformer

\section{INTRODUCTION}

The emerging $5 \mathrm{G}$ cellular systems are looking at mmWave frequencies to augment the available spectrum and to provide high data rates for both line-of-sight (LOS) and non-lineof-sight (NLOS) wireless channels. The inherent high pathloss in mmWave frequency bands limits the range and hence the coverage of any mmWave deployment. However, these coverage issues can be addressed by smart beamforming and steering radiation patterns towards the intended user. Phased array antennas are intended to produce highly focused directive single beams that can be scanned electronically[1]. However, it is also possible to generate multiple and shaped beams from the same aperture. Several antenna arrays with multiple beam capability are discussed in the literature. Multiple beams can be realized from a single aperture with multi-feed reflector systems[2], lens-based design[3], and switched beam approaches [4]-[6]. Skolnik[7] has proposed a method to generate two simultaneous beams from a continuous line source, where the combined current distribution of the line source for two beams is computed by summing the complex current distributions of the two independent beams.

Many analytic, iterative and optimization methods [8]-[11] are proposed for specific shaped beam synthesis patterns and demonstrated on a linear array. Woodward Lawson synthesis method [12] relies on arranging suitable multiple field distributions over an aperture and superimposing them to realize

S. Prasad, and P.H Rao are with Microwave Communication and Antennas Division (MCAD), SAMEER-CEM, Chennai-600113, India. (e-mail: s.prasad@cem.sameer.gov.in, phrao@cem.sameer.gov.in)

M. Meenakshi is with Department of Electronics and Communication Engineering, CEG, Anna University, Chennai-600025, India. (e-mail: meena68@annauniv.edu) desired shaped beam but without any control on the sidelobe levels outside the shaped beam. Stutzman[9] introduced a successive iteration method by adding series of corrective patterns to get the required pattern. Elliott and Orchard[8], [13] demonstrated a shaped beam synthesis by displacing the roots on the polynomial representation of the sum pattern. Certain roots are displaced to fill the null region to get a shaped low ripple pattern, and these demonstrations are specific to flattopped and $\csc ^{2} \theta \cos \theta$ patterns. Yuen T. Lo[14] suggested a linear transformation of field excitation, where the field is expressed in terms of a set of patterns, and each pattern represents a uniform aperture excitation. Li et al.[15] proposed GA-based shaped beam synthesis using superposition principle and Taylor method with control on sidelobe levels beyond shaped beam.

All these approaches are limited to multiple beams over fixed directions and do not support dynamically changeable shaped beams. A new synthesis and computation method is presented in this paper to generate any user-specified multiple beams and shaped beams in any arbitrary 3D space. This method is simple and easy to implement both in linear and planar arrays and it is capable of steering the multiple beams independently to any arbitrary angle in $\theta, \phi$ with equal and unequal power levels. This method is extended to synthesize any arbitrary shaped beam with optimally placed multiple beams at appropriate locations with specific power ratios. The proposed method is practically demonstrated on a fully functional integrated $5 \mathrm{G}$ mmWave tiled phased array in hybrid beamforming architecture. In a practical large-scale 5G antenna deployment, hybrid beamforming is the suitable architecture. This architecture supports multiple data streams and multiple numbers of shaped beams with less complexity, cost, and power consumption [16]. The shaped beam patterns are practically implemented on an $8 \times 8 \mathrm{mmWave}$ phased array [17], [18] which can produce scannable single, dual, multiple, and shaped beams in $(\theta, \phi)$ from the same hardware.

The proposed computation method is more efficient to implement in evolutionary algorithms to calculate the antenna element weights for controllable side lobe level. Modified differential evolution (MDE) algorithm is used in this paper, to calculate the optimum number of independent beams and their corresponding locations in $(\theta, \phi)$ to get standard and userdefined shaped beams. It uses only the peaks and their power as variables to generate shaped beam patterns irrespective of the array size.

The paper is organized as follows. The design of the $5 \mathrm{G}$ mmWave phased array is discussed in section II. A computation method to generate multiple beams from a single aperture is given in Section III. Shaped beam synthesis using multiple beams is explained in section IV. Calculation of shaped beam 
amplitude and phase weights using an evolutionary algorithm is presented in section V. The measured results for multiple and shaped beams are presented in section VI followed by conclusions in section VII.

\section{DESIGN OF A 5G mmWAVE PLANAR PHASED ARRAY ANTENNA}

In an electronically scanned phased array, beamforming is achieved by independently controlling the amplitude and phase of each antenna element using beamformer chips [19], [20]. These chips are intended to change the amplitude and phase values within microseconds for fast scanning. Initially, a single antenna element is designed for $5 \mathrm{G}$ at $28 \mathrm{GHz}$ with $400 \mathrm{MHz}$ bandwidth on multilayer stackup and optimized using 3-D full-wave electromagnetic simulation software, ANSYS HFSS [21]. A $2 \times 2$ array with a quad-channel beamformer is utilized as a unit cell of larger planar phased array configurations. Sixteen such beamformers are used to design a 64-element $(8 \times 8)$ phased array. Independent four RF vertical interconnect access (VIA) holes connect each quad beamformer chip and four microstrip patch antennas. Parasitic patches are placed around the active antenna elements to create a uniform environment for the edge elements of the planar array. A printed cavity configuration sandwiched between the ground layer and the patch layer helps to reduce mutual coupling between radiating elements.

TABLE I

PHASED ARRAY ANTENNA SPECIFICATIONS

\begin{tabular}{lll}
\hline \hline & Parameter & Value \\
\hline 1. & Operating frequency & $28 \mathrm{GHz}$ \\
\hline 2. & $3 \mathrm{~dB}$ bandwidth & $>400 \mathrm{MHz}$ \\
\hline 3. & Array size & $8 \times 8,64$ element with parasitic patches \\
\hline 4. & Element spacing & $0.65 \lambda$ \\
\hline 5. & Antenna element gain & $4 \mathrm{~dB}$ \\
\hline 6. & Antenna feed type & Probe feed \\
\hline 5. & Antenna array gain & $24 \mathrm{~dB}$ \\
\hline 6. & Transmit EIRP & $+56 \mathrm{dBm}$ (peak) \\
\hline 7. & Receiver noise figure & $6.3 \mathrm{~dB}$ per channel \\
\hline 8. & Polarization & Single, Linear \\
\hline 9. & Amplitude resolution & $0.4 \mathrm{~dB}$ \\
\hline 10. & Phase resolution & $5.625^{\circ}$ \\
\hline 11. & Scanning steps & $1.43^{\circ}$ \\
\hline & &
\end{tabular}

The array is optimized by considering the selection of substrate material, the number of substrate layers, type of antenna element, feeding mechanism, electrical, mechanical, and thermal design. Grounded coplanar waveguide (GCPW) transmission lines on the bottom layer of the printed circuit board (PCB) along with miniaturized surface mounted power dividers, distribute the input RF signal equally to all the beamformer chips. The input RF signal from a TR (trans receive) module is distributed to all 16 beamformers using 15 two-way power dividers. A fully integrated 5G mmWave planar phased array antenna, as shown in Fig.1a is realized on a 10 Layer substrate with an overall PCB thickness of $2.5 \mathrm{~mm}$. The top three layers are used for antenna elements realization.
The bottom layer is populated with components which include the beamformer chips, power distribution network, the biasing circuits. The remaining middle layers are used for power supply and digital control lines.
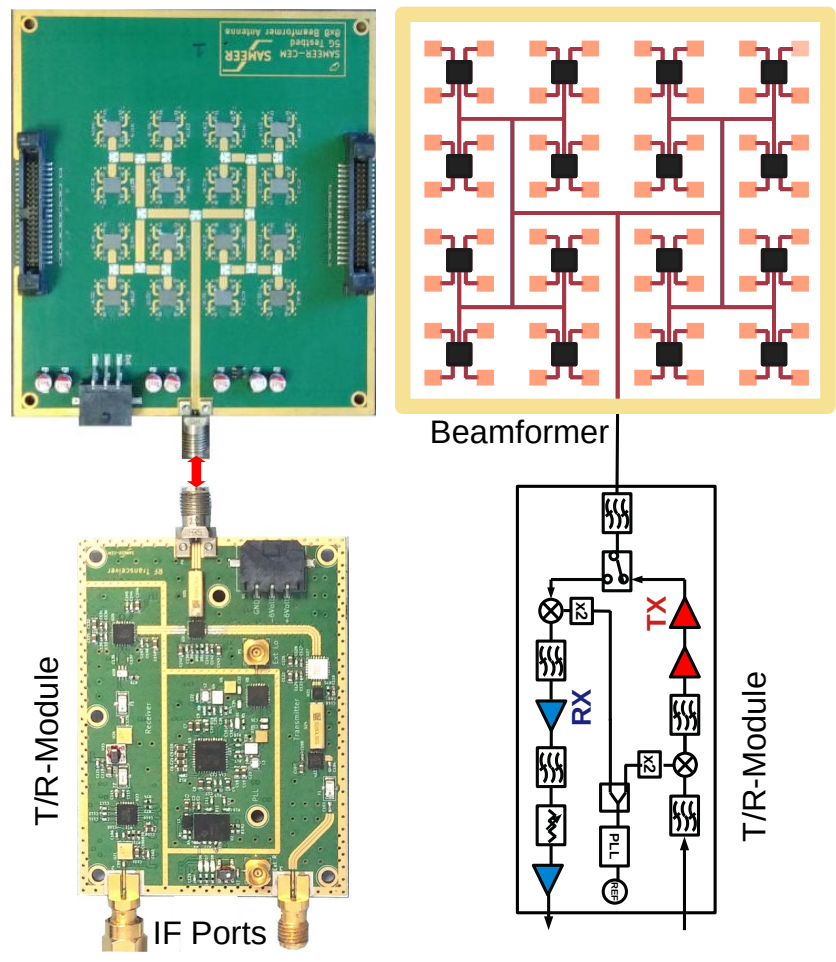

Beamformer

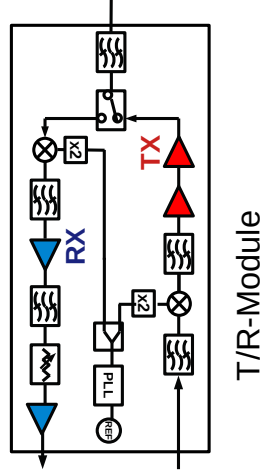

(a)

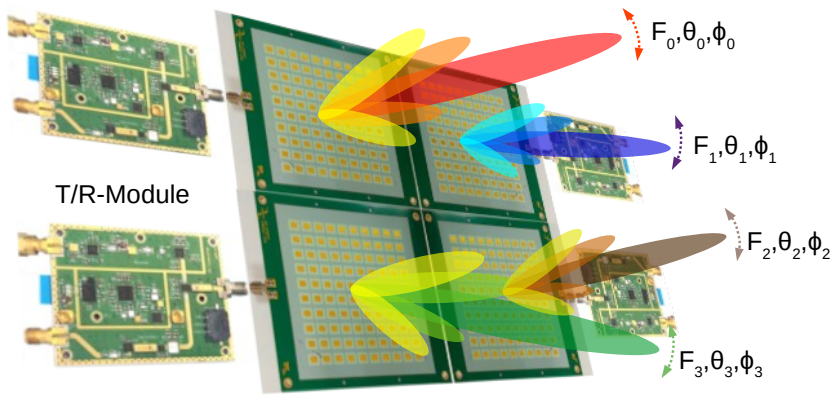

(b)

Fig. 1. Fully integrated $8 \times 85 \mathrm{G} \mathrm{mmWave} \mathrm{antenna} \mathrm{array.} \mathrm{(a)} \mathrm{Antenna} \mathrm{array}$ with TR module. (b) Phased array antenna in $2 \times 2$ hybrid arrangement.

The TR module is a single-stage up/down converter with $3.5 \mathrm{GHz}$ as intermediate frequency (IF). An onboard programmable PLL operating at $12.25 \mathrm{GHz}$ acts as a local oscillator (LO) for Tx and Rx mixers. The mixers internally multiply the LO to get $24.5 \mathrm{GHz}$ for generating the required $28 \mathrm{GHz} \mathrm{RF}$. The mixers are followed by RF filter in Tx and IF filter in Rx modes to allow only the required signals and block all the interferences. A programmable attenuator is included in the receiver chain to protect the baseband ADC from saturation and hence increase the dynamic range. The transmitter and receiver chains are connected to a single pole double throw (SPDT) high isolation switch in time division duplex(TDD) mode. The transceiver module has an optional external LO, 
external PLL reference input to synchronize multiple T/R modules in hybrid beamforming. Fig.1b shows the proposed $2 \times 2$ hybrid beamformer with four $8 \times 8 \mathrm{mmWave}$ phased array antennas and respective $\mathrm{T} / \mathrm{R}$ module to produce four single beams or multiple beams patterns to any arbitrary angle $\left(F_{n}\right.$, $\left.\theta_{n}, \phi_{n}\right)$.

\section{MultiBEAm COMPUTATION}

Conventional passive switched beam antennas based on Butler matrix and Rotman lens can produce multiple beams when all the input ports are simultaneously excited. However, the beams are limited to a fixed direction and are non-steerable. Woodward [12] reported that the superposition of narrow beams produces a combined pattern of the individual beams. It is possible to arrange these narrow beams to get standard and user-defined shaped beams. We propose a computation method derived from the array factor calculations to obtain weights for arbitrarily shaped patterns with low sidelobe levels using this multibeam principle. These beams are programmed to radiate towards any arbitrary locations with different power ratios, both in azimuth and elevation angles. Scanning the shaped beam alters the beam pattern due to scan loss. The beam peaks are adjusted to maintain the beam shape with prior knowledge of the phased array pattern. The following subsection presents the computation for generating multiple beams.

\section{A. Array factor for single beam}

The output of $N$ element antenna array for an input signal $s(t)$ is given by,

$$
y(t)=s(t) f(\boldsymbol{k}) \underbrace{\left[\sum_{n=1}^{N} w_{n} e^{-j \boldsymbol{k} \mathbf{d}_{n}}\right]}_{A F(\theta, \phi)} .
$$

where,

$$
\boldsymbol{k}=\left(k_{x}, k_{y}, k_{z}\right)=\frac{2 \pi}{\lambda}(\sin \theta \cos \phi, \sin \theta \sin \phi, \cos \theta) .
$$

The output is a function of element factor $f(\boldsymbol{k})$ and array factor $A F(\theta, \phi)$, multiplied by the input RF signal $s(t)$.

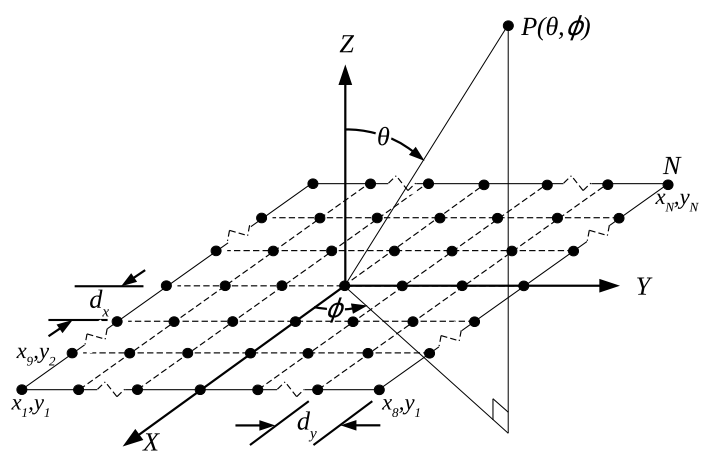

Fig. 2. Two dimensional $\mathrm{N}$ element planar square array element placement. $d_{x}$ and $d_{y}$ are the element spacing in the planar grid.

The array factor of a two dimensional $\mathrm{N}$ element array towards the direction $P$ as shown in Fig.2 is given by [22],

$$
A F(\theta, \phi)=\sum_{n=1}^{N} w_{n} e^{-j \frac{2 \pi}{\lambda}\left(x_{n} \sin (\theta) \cos (\phi)+y_{n} \sin (\theta) \sin (\phi)\right)},
$$

where,

$$
\begin{aligned}
& w_{n}=A_{n} e^{j\left(\beta_{x n}+\beta_{y n}\right)}, n=0,1, \ldots, N, \\
& \beta_{x n}=\frac{2 \pi}{\lambda}\left(x_{n} \sin \left(\theta_{d}\right) \cos \left(\phi_{d}\right)\right), \\
& \beta_{y n}=\frac{2 \pi}{\lambda}\left(y_{n} \sin \left(\theta_{d}\right) \sin \left(\phi_{d}\right)\right) .
\end{aligned}
$$

where $A_{n}$ is the amplitude taper across the array elements to control the side lobe level from the main beam. $w_{n}$ denotes the amplitude and phase of the $n$th antenna element, and the element location is described by a vector $\mathbf{d}_{n}=\left[x_{n}, y_{n}, z_{n}\right]$. $x_{n}, y_{n}$ are the positions of the antenna elements based on the inter element spacing $d_{x}, d_{y}$ respectively. $\beta_{x n}$ and $\beta_{y n}$ are the progressive phases in $x$-, $y$ - directions to steer the beam towards the desired direction $\left(\theta_{d}, \phi_{d}\right)$.

A generalized array factor for a $N$ element planar phased array with arbitrary beam steering is given by,

$$
\begin{array}{r}
A F(\theta, \phi)=\sum_{n=1}^{N} A_{n} e^{-j \frac{2 \pi}{\lambda}\left(x_{n} \sin (\theta) \cos (\phi)+y_{n} \sin (\theta) \sin (\phi)\right)} \\
e^{j\left(\beta_{x n}+\beta_{y n}\right)} .
\end{array}
$$

\section{B. Computation of array factor in linear and planar array for multiple beams}

1) Line source configuration: Independent multiple beams can be generated with a single beamformer by modifying the amplitude and the phase at each element of the aperture. The relation for a two-beam aperture distribution on a continuous line source is given in [7].

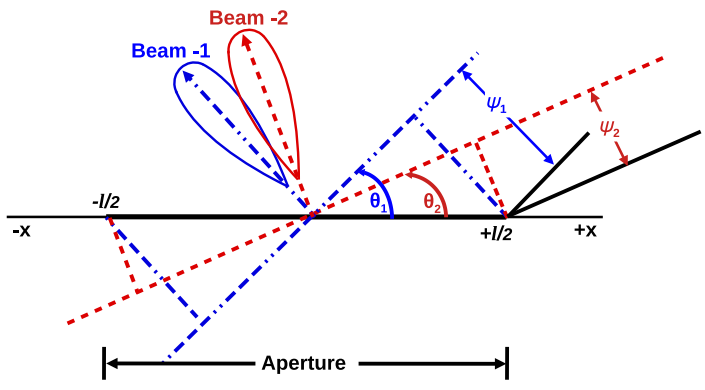

Fig. 3. Aperture distribution for two beams on a line source.

Two independent beams with same amplitude distribution $F(x)$ along the $x$ - axis and inclined phase fronts $\psi_{1}, \psi_{2}$ are presented in Fig.3. Here $\psi_{1}, \psi_{2}$ are the phase fronts along the aperture edges for the two beams respectively. The combined aperture excitation on a continuous line source for the two beam peaks with unequal power is given by,

$$
F(x, \psi)=F(x) e^{j 2 \psi_{1}\left(\frac{x}{l}\right)}+a F(x) e^{j 2 \psi_{2}\left(\frac{x}{l}\right)} .
$$

Where $F(x, \psi)$ is the aperture distribution along the continuous line source between $-l / 2$ to $+l / 2$ and ' $a$ ' is the ratio of the two beam peak amplitudes. From (6),

$$
\begin{aligned}
& F(x, \psi)=F(x)\left[(1+a) \cos \left(\left(\psi_{1}-\psi_{2}\right)\left(\frac{x}{l}\right)\right)\right. \\
& \left.\quad+j(1-a) \sin \left(\left(\psi_{1}-\psi_{2}\right)\left(\frac{x}{l}\right)\right)\right] e^{j\left(\psi_{1}+\psi_{2}\right)\left(\frac{x}{l}\right)} .
\end{aligned}
$$


If both beam peaks have same amplitude, then $a=1$, which gives,

$$
F(x, \psi)=2 F(x) \cos \left[\left(\psi_{1}-\psi_{2}\right)\left(\frac{x}{l}\right)\right] e^{j\left(\psi_{1}+\psi_{2}\right)\left(\frac{x}{l}\right)}
$$

For a two-beam case with the same amplitude distribution and differently inclined phase fronts, the effective aperture amplitude distribution varies sinusoidally and the phase distribution is the resultant of two inclinations $\psi_{1}$ and $\psi_{2}$ which varies periodically from 0 to $\pi$.

2) Planar source configuration: A $2 \mathrm{D}$ plane extending from $-l_{x} / 2$ to $+l_{x} / 2$ in $x$ direction and $-l_{y} / 2$ to $+l_{y} / 2$ in $y$ direction is shown in Fig.4. Here $\left(\psi_{1 x}, \psi_{1 y}\right)$ and $\left(\psi_{2 x}\right.$, $\psi_{2 y}$ ) are the inclined phase fronts at the aperture edges in $x$ and $y$ directions for any two arbitrary beams towards $\left(\theta_{1}\right.$, $\left.\phi_{1}\right)$ and $\left(\theta_{2}, \phi_{2}\right)$. The combined excitation on the aperture of

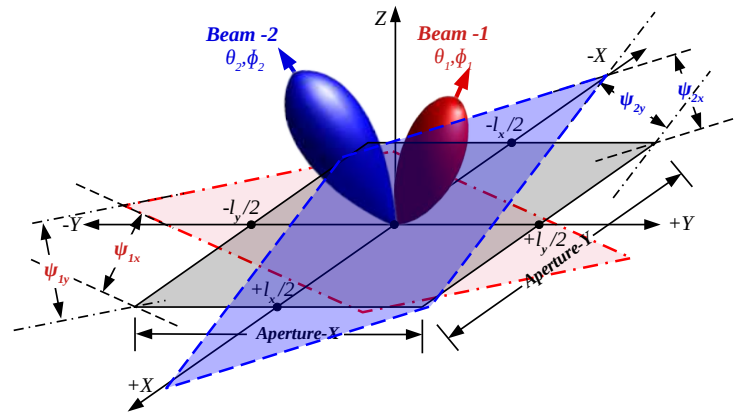

Fig. 4. Aperture distribution for two beams on a planar source.

the uniform planar source for two arbitrary beams with equal amplitude distribution $F(x, y)$ is given by,

$$
\begin{aligned}
& F\left(x, \psi_{x}, y, \psi_{y}\right)=2 F(x, y) \cos \left[\left(\left(\psi_{1 x}-\psi_{2 x}\right)\left(\frac{x}{l}\right)\right.\right. \\
& \left.\left.+\left(\psi_{1 y}-\psi_{2 y}\right)\right)\left(\frac{y}{l}\right)\right] e^{j\left(\left(\psi_{1 x}+\psi_{2 x}\right)\left(\frac{x}{l}\right)+\left(\psi_{1 y}+\psi_{2 y}\right)\left(\frac{y}{l}\right)\right)} .
\end{aligned}
$$

As in the linear array case of (8), the planar aperture distribution (9) shows that the effective amplitude distribution varies sinusoidally while the phase is the resultant of two inclinations in $x$ - and $y$ - planes. This clearly signifies the importance of both amplitude and phase control for simultaneous multibeam configuration.

The general array factor for a continuous rectangular plane between $-l_{x} / 2$ to $+l_{x} / 2$ and $-l_{y} / 2$ to $+l_{y} / 2$ is given by,

$$
\begin{aligned}
& A F(\theta, \phi)=\int_{-l_{x} / 2}^{+l_{x} / 2} \int_{-l_{y} / 2}^{+l_{y} / 2} F\left(x, \psi_{x}, y, \psi_{y}\right) \\
& {\left[e^{-j \frac{2 \pi}{\lambda}(x \sin (\theta) \cos (\phi)+y \sin (\theta) \sin (\phi))}\right] d x d y . }
\end{aligned}
$$

here $F\left(x, \psi_{x}, y, \psi_{y}\right)$ as in (9) is the continuous aperture distribution required to steer the beam towards the desired direction.
3) Computation of weights: The array factor $A F(\theta, \phi)$ of (10) can be extended to a discrete planar array with $N$ elements. The discrete multibeam array factor is expressed as,

$$
\begin{aligned}
A F(\theta, \phi) & =\sum_{n=1}^{N} W_{n} e^{-j \frac{2 \pi}{\lambda}\left(x_{n} \sin (\theta) \cos (\phi)+y_{n} \sin (\theta) \sin (\phi)\right)}, \\
W_{n} & =a_{1} w_{n}\left(\theta_{1}, \phi_{1}\right)+a_{2} w_{n}\left(\theta_{2}, \phi_{2}\right)+\ldots
\end{aligned}
$$

where $W_{n}$ is the total aperture distribution calculated by combining the weights of individual beams $\left(w_{n}\right)$ and $a_{1}, a_{2} \ldots a_{K}$ are the required ratios of the multiple beams peak amplitudes for unequal beam pattern.

$$
\begin{aligned}
W_{n} & =\sum_{k=1}^{K} a_{k} w_{n}\left(\theta_{k}, \phi_{k}\right), \\
w_{n} & =A_{n} e^{j \frac{2 \pi}{\lambda}\left(x_{n} \sin (\theta) \cos (\phi)+y_{n} \sin (\theta) \sin (\phi)\right)} .
\end{aligned}
$$

for multibeam case the amplitude taper $A_{n}$ across the elements are considered to be unity then the combined weights for $K$ beams is given by,

$$
W_{n}=\sum_{k=1}^{K} a_{k} e^{j \frac{2 \pi}{\lambda}\left(x_{n} \sin \left(\theta_{k}\right) \cos \left(\phi_{k}\right)+y_{n} \sin \left(\theta_{k}\right) \sin \left(\phi_{k}\right)\right)},
$$

where,

$n-0,1, . ., N$,

$K$ - number of beams required,

$a_{k}$ - power ratio of $k$ th beam,

$\theta_{k}$ - beam direction in $\theta$ angle for $k$ th beam,

$\phi_{k}$ - beam direction in $\phi$ angle for $k$ th beam.

One of the common requirements for an array design is a low Side Lobe Level (SLL). Antenna pattern synthesis with Taylor windowing, which is having selectable side lobe level control [22] with minimum main beam broadening is implemented in this paper. The Taylor weights $\left(T_{n}\right)$ can be computed using the procedure described by Elliot [23]. The amplitude weights across the elements $\left(A_{n}\right)$ are replaced with the Taylor weights $T_{n}$ to reduce the SLL. The Taylor synthesized multibeam pattern weights are given by,

$$
W_{T_{n}}=T_{n} \sum_{k=1}^{K} a_{k} e^{j \frac{2 \pi}{\lambda} \sin \left(\theta_{k}\right)\left(x_{n} \cos \left(\phi_{k}\right)+y_{n} \cos \left(\phi_{k}\right)\right)} .
$$

The resultant array factor of a taylor weighted multibeam array with unequal peak is

$$
A F(\theta, \phi)=\sum_{n=1}^{N} W_{T_{n}} e^{-j \frac{2 \pi}{\lambda}\left(x_{n} \sin (\theta) \cos (\phi)+y_{n} \sin (\theta) \sin (\phi)\right)} .
$$

\section{MUlTi BEAM AND SHAPED BEAM SYNTHESIS}

The method described in section III is applied on a typical planar $16 \times 16$ element phased array with $0.5 \lambda$ inter-element spacing in $x, y$ directions. The number of beams, along with their peak values, beam pointing angles, and the desired side lobe level (SLL) are given as inputs to the computation method 
to generate user-defined multiple beams. Various use cases including multi beams and shaped beams are synthesized and validated with the proposed computation method. The aperture weights calculated from Eqn.14 are quantized for six bits and applied to the individual elements of the array to generate multiple beams and shaped beams. The six-bit quantization is chosen to replicate the practical beamformer chip configuration used in the practical realization of the phased array.

\section{A. Dual beam in Azimuth $(\phi)$ angle}

A typical near and far multiuser deployment scenario from a base station is synthesized by generating dual-beam pattern towards the intended users. Fig.5a shows a comparison of a single beam and a dual-beam directed at $+20^{\circ}$ and $-20^{\circ}$ with equal power. A difference of $3 \mathrm{~dB}$ in the peak power level is evident when the single beam is split into dual beams while maintaining the required sidelobe level. Fig 5b shows two cases of dual-beam patterns with unequal peak power levels scanned to symmetrical and asymmetrical azimuth angles. In the symmetrical case, the dual-beam peaks are scanned to $+20^{\circ}$ and $-20^{\circ}$ with a $5 \mathrm{~dB}$ peak power difference between the two peaks. In the asymmetrical case, the dual-beam peaks are scanned to $0^{\circ}$ and $15^{\circ}$ with a peak power difference of $4 \mathrm{~dB}$.

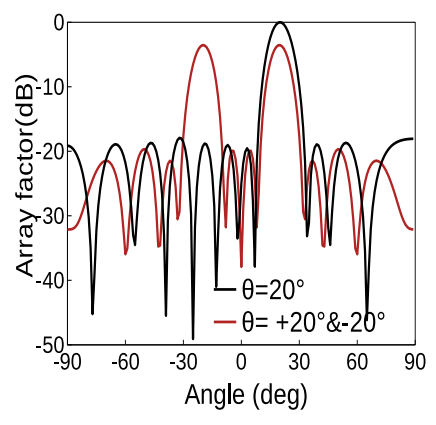

(a)

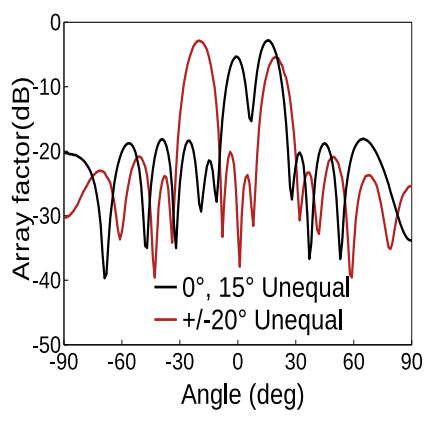

(b)
Fig. 5. Multiuser with equal and unequal power scenario. (a) Equal power dual beam pattern at $-20^{\circ}$ and $+20^{\circ}$. (b) Taylor weighted unequal gain beam pattern at $\pm 20^{\circ}$ and $0^{\circ}, 15^{\circ}$.

\section{B. Dual and multibeam in azimuth-elevation $(\theta, \phi)$ angles}

The proposed computation method is used to generate dual beams and multibeams pointing at any user defined arbitrary angles in $\theta, \phi$ and are shown in Fig.6. Fig.6a shows a 20dB SLL taylor distribution weighted multibeam pattern with seven beam peaks in $\theta, \phi$. The center beam is at $\theta=0^{\circ}$ and $\phi=0^{\circ}$ direction and the remaining six beams are at $\theta=30^{\circ}$ and $\phi=0^{\circ}, 60^{\circ}, 120^{\circ}, 180^{\circ}, 240^{\circ}, 300^{\circ}$ respectively.

All the beam peaks are maintained at the same power level. Here the peak power is equally shared between multiple beams. The multibeam computation method is capable of scanning either one of the beam peaks or multiple beam peaks to any predefined angle in $\theta, \phi$. Fig.6b shows another use case of two unequal beam peaks directed towards $\theta=35^{\circ}, \phi=0^{\circ}$

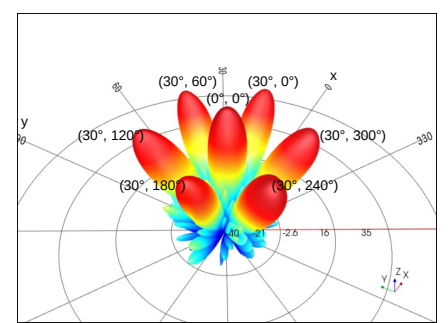

(a)

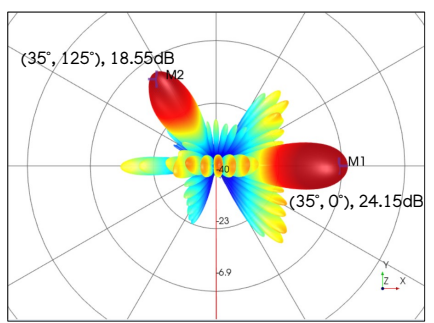

(b)
Fig. 6. Multiple beams at predefined angles $(\theta, \phi)$. (a) Seven simultaneous beam with equal power. (b) Two arbitrary beam peaks with unequal power level.

and $\theta=35^{\circ}, \phi=125^{\circ}$. These patterns are generated with a power ratio of $1,0.3$ ( $5 \mathrm{~dB}$ power difference) to serve the users at various distances from the gNodeB of $5 \mathrm{G}$ base station. This unequal beam power pattern in $\theta, \phi$ enables optimized coverage and throughput for the users separated in various floors of a multilevel building.

\section{Synthesis of shaped beam from multiple beams}

The proposed multi-beam computation of section III is extended to synthesize shaped beams by generating and optimizing multiple beam peaks and their locations. For an array of length $L=(N-1) d_{x}$, it is possible to generate a $N$ number of beam lobes [1]. Primary and secondary maxima locations $\left(\theta_{p}\right)$ are defined by the following equation,

$$
\theta_{p}=\sin ^{-1}\left(\frac{2 i}{N}\right)
$$

for $i= \pm 1 / 2, \pm 3 / 2 \ldots \pm(N-1) / 2$ for $N$ Even and $i=$ $0, \pm 1, \pm 2 \ldots \pm(N-1) / 2$ for $N$ Odd. Two typical shaped beams viz. sector beam and $c s c^{2}$ pattern are considered to validate the proposed method. Four independent beams with equal power towards $\theta=-22^{\circ},-7.2^{\circ}, 7.2^{\circ}, 22^{\circ}$ and their combined pattern are shown in Fig.7a. These beam peaks generate a sector beam pattern. However, the inband ripples are noticeable. To make a proper shaped beam the gaps between these ripples have to be filled with additional beams to make a response with minimum ripples. Hence the term $i$ is modified to $i=0, \pm 1, \pm 2 \ldots \pm N / 2$ beams. By adding more beam peaks in between the ripples, a flat beam with less than $0.2 \mathrm{~dB}$ ripple can be achieved. Initially, the beam peaks are placed into the predefined locations with equal power. A flat response shown in Fig.7b is achieved by gradually adjusting the appropriate peak power.

Similarly, a cosecant pattern is generated by starting with three peaks to cover $50^{\circ}$ phase angle (Fig.8a). The peak power is reduced to $1,0.75,0.5,0.25$ power ratios to get the required beam as shown in Fig.8b. The additional peak angles and their power are adjusted to get a low ripple cosecant pattern and the steps are given in Fig.8.

One of the primary functions of a phased array is beam steering. It is also possible to steer the shaped beam without affecting the shaped pattern by introducing a progressive phase between the elements on top of the existing phase weights. The 


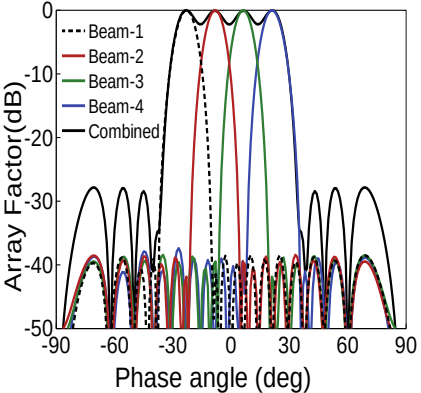

(a)

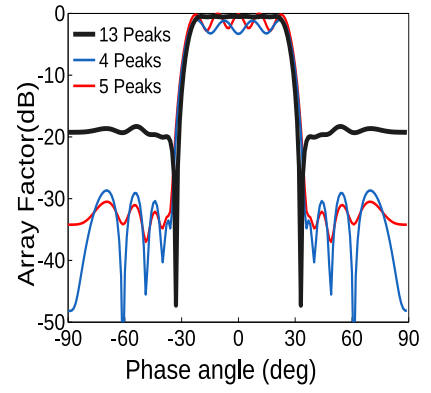

(b)
Fig. 7. Low ripple sector beam generation from multiple beam peaks. (a) Sector beam with four independent equal power beams. (b) Sector beam pattern for $4,5,13$ peaks.

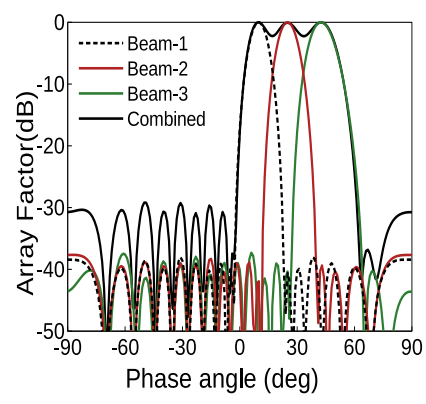

(a)

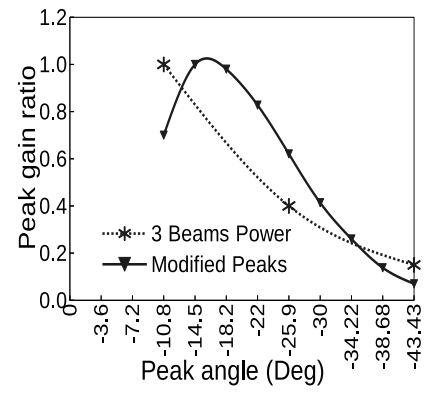

(c)

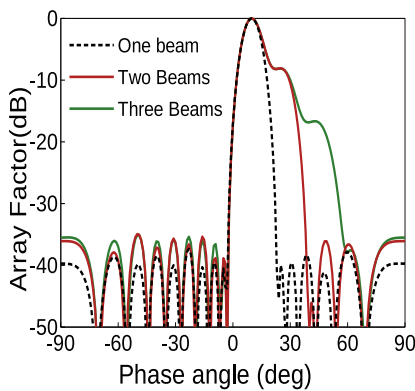

(b)

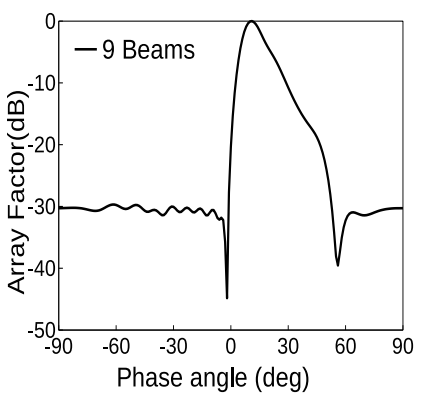

(d)
Fig. 8. $c s c^{2}$ and low ripple $c s c^{2}$ beam pattern generation from multiple beam peaks. (a) Sector beam with three independent equal power beams. (b) Radiation pattern after progressively adjusting the peak power. (c) Peak gain adjustment for additional peaks to get a low ripple pattern. (d) Low ripple $c s c^{2}$ beam pattern with 13 independent peaks.

amplitude weights remain unchanged here. For an $\mathrm{N}$ element array, the updated phase weight is given by,

$$
w_{n}\left(\theta_{m}\right)=w_{n}(\theta+n \beta)
$$

where $\beta$ is the progressive phase required to steering beam towards $\theta_{m}$ direction from the current position. $w_{n}(\theta)$ is the phase weight of $n^{\text {th }}$ element. Fig.9 shows the $c s c^{2}$ and broad beam patterns swept across $40^{\circ}$ angle.Observation from Fig.9 is that the shape of the beam remains the same during scanning.

\section{SYNTHESIS OF SHAPED BEAM USING EVOLUTIONARY ALGORITHM}

Beam steering and beam shaping applications use numerical calculations or evolutionary algorithms to find the weights

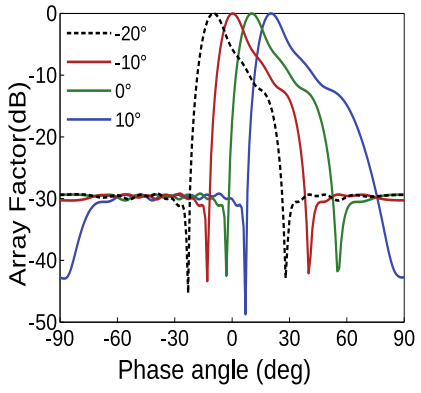

(a)

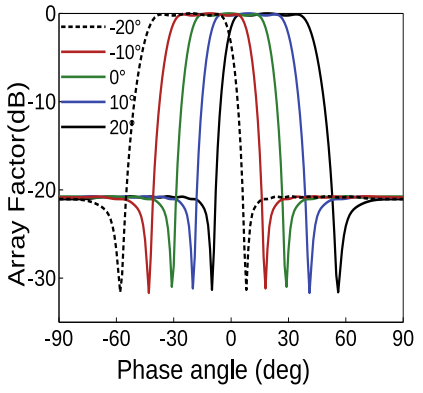

(b)
Fig. 9. Shaped beam steering using the progressive phase control. (a) $\pm 20^{\circ}$ scanning of the cosecant square beam. (b) Broadbeam steering between $\pm 20^{\circ}$.

of the array elements. The numerical calculations are often limited to specific beam shapes, and the evolutionary algorithms are more optimal for shaped beam generation. The evolutionary algorithms optimize the excitation amplitude and phase of each element to obtain the required beam pattern [24]. However, the time taken by the evolutionary process depends on the number of variables (parents) in the calculation. The proposed multi-beam computation can simplify the convergence of the algorithm by reducing the number of variables. Here the number of beam peaks, direction, power of each beam peak, and SLL are sufficient to obtain the required beam shape irrespective of the array size. Modified differential evolution (MDE) algorithm is used here to optimize the beam peaks using the variables specified. The array factor is given by,

$$
\begin{aligned}
A F(\theta) & =\sum_{n=1}^{N} W_{T n} e^{-j \frac{2 \pi}{\lambda} x_{n} \sin (\theta)} \\
W_{T n} & =T n \sum_{k=1}^{K} a_{k} e^{j \frac{2 \pi}{\lambda} x_{n} \sin \left(\theta_{k}\right)}
\end{aligned}
$$

$K, a_{k}, \theta_{k}, S L L$ of $T n$ are the variable used in the optimization algorithm. Number of variables for the algorithm are irrespective of the size of the array. The fitness function to be minimized is defined as,

$$
\begin{aligned}
& \text { Fitness }=\sum_{i=-90}^{90}\left|A F\left(\theta_{i}\right)_{\text {req }}-A F\left(\theta_{i}\right)_{\text {opti }}\right| \\
& \text { where } \\
& \quad \forall A F\left(\theta_{i}\right)_{\text {opti }}<A F\left(\theta_{i}\right)_{\text {req }}<-30 d B, \text { Fitness }=0 .
\end{aligned}
$$

The fitness function minimizes the error between the required user-defined far-field pattern $A F(\theta)_{r e q}$ with the optimized radiation pattern $A F(\theta)_{\text {opti }}$ for angles between $-90^{\circ}$ to $+90^{\circ}$. The user and optimized pattern for different shapes are shown in Fig. 10.

The fitness function takes only the normalized array factor values above $-30 \mathrm{~dB}$ for faster convergence. The MDE algorithm calculates the number of beams, their angle, power level, and the SLL required to generate the user-defined pattern for a 16 element linear array. Fig.10 shows the optimized and normalized array factor plots. Dual-beam in Fig.10a is made with two unequal beams towards $\theta=15^{\circ},-20^{\circ}$ with power 


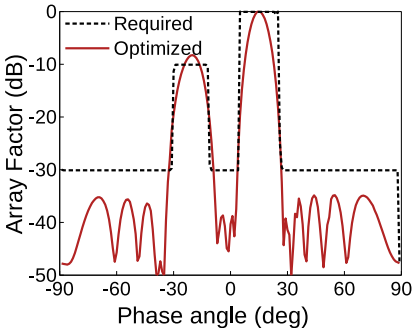

(a)

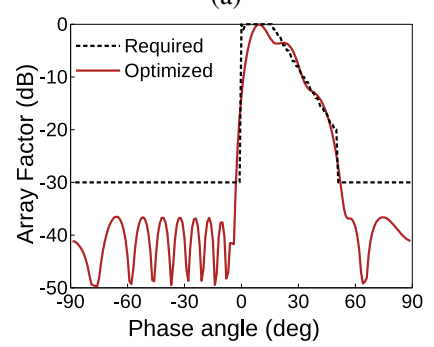

(c)

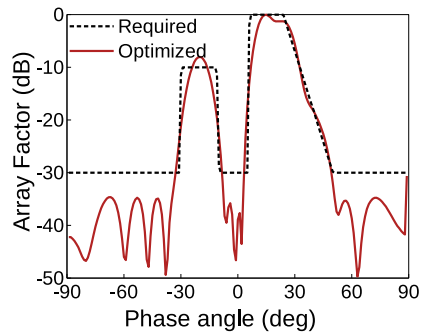

(b)

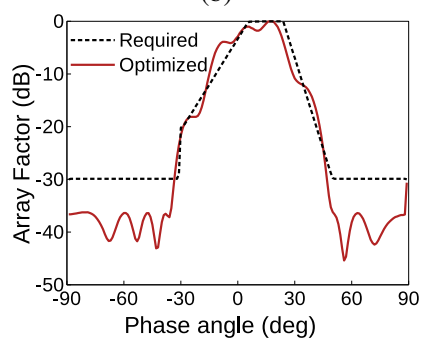

(d)
Fig. 10. Optimized shaped beam Pattern on a 16 element array using DE algorithm. (a) Dual unequal beam. (b) Dual shaped beam. (c) Cosecant squared beam. (d) User defined shaped beam.

ratios of 1 and 0.4 . The figures also show the optimized SLL for the specified bandwidth. Fig.10b shows another dual-beam where the second beam is made broad. Fig.10c shows a cosecant squared pattern using three-beam peaks. Fig.10d shows an arbitrary shape beam generated using five beam peaks optimized by the MDE algorithm. The software calculated values are listed in Table. II for different types of beam patterns shown in Fig.10.

TABLE II

MDE CALCULATED BEAM PEAK VALUes $(\theta$, PEAK RATio, SLL $d$ B $)$

\begin{tabular}{lcccccc}
\hline \hline Type & Beam1 & Beam2 & Beam3 & Beam4 & Beam5 & SLL \\
\hline Dual unequal & $15^{\circ}, 1.0$ & $-20^{\circ}, 0.4$ & - & - & - & -34.8 \\
Dual shaped & $37^{\circ}, 0.13$ & $22^{\circ}, 0.89$ & $16^{\circ}, 1.00$ & $-20^{\circ}, 0.29$ & - & -44.4 \\
$\csc c^{2}$ pattern & $10^{\circ}, 1.00$ & $22^{\circ}, 0.64$ & $37^{\circ}, 0.18$ & - & - & -42 \\
User defined & $34^{\circ}, 0.25$ & $18^{\circ}, 1.00$ & $4.9^{\circ}, 0.96$ & $-8.3^{\circ}, 0.64$ & $-24^{\circ}, 0.13$ & -43.4 \\
\hline
\end{tabular}

\section{Vi. Measurement and Validation}

Pattern measurement for the shaped beam uses the weights calculated from the shaped beam generation method explained in section III. Alternatively, the MDE algorithm also can be used to calculate the amplitude and phase weights from the optimized peaks, their angle, and the power ratio. A PCbased software loads the quantized weights (for the 6-bit beamformer) to the mmWave phased array.

Fig.11 shows the compact area test range (CATR) setup used for the radiation pattern measurement. CATR is an antennaspecific test and measurement facility to verify antenna performance. CATR provides a far-field radiation pattern inside an anechoic chamber over a small area. It uses a primary antenna and a secondary reflector that works together to generate a region of plane waves resulting in a quiet zone

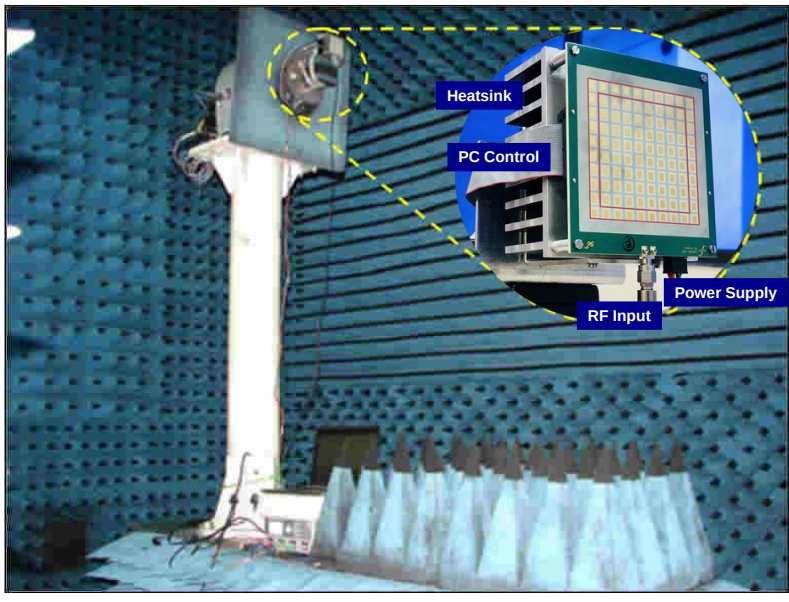

Fig. 11. Compact area test range (CATR) measurement setup.

volume. The testing antenna has to be placed in a quiet zone for measurement. The antenna unit with heat sink and controller board is mounted on a rotating nonmetallic mast. The limitation of the CATR measurement setup is that the antenna measurement has to be carried out only in receive mode. The phased array antenna is mounted on the mast after the initial calibration of CATR. The weights for single, dual, unequal, sector, sector beam with null and $\csc ^{2}$ patterns are loaded and measured. Fig. 12 is the comparison of the measured patterns with the simulated results. It is important to note that the antenna array calibration is not been done to compensate for the phase and amplitude variations.

Figs.12a,12b shows the single beam pattern steered towards the $+25^{\circ}$ and $-25^{\circ}$ angles. Here the measurement is precisely matching the simulated results towards the peak. The side lobes are closely following the simulations. Fig.12c is the dualbeam pattern with peaks directed towards $\pm 25^{\circ}$ in the azimuth plane. The radiation pattern has two peaks with equal power levels. In Fig.12d, one of the peak power is reduced to make it unequal. Here the measurement is closely following the simulation. The minor power difference is due to the missing calibration. The multibeam pattern measurement is further extended into complex shapes. Fig.12e shows the sector beam pattern that covers the $-25^{\circ}$ to $+25^{\circ}$ range. A ripple level less than $1 \mathrm{~dB}$ is observed in the measured response. Fig.12f shows the $\csc ^{2}$ pattern generated by placing three beams next to each other with decrementing power levels. The plot shows that the side lobes are below $15 \mathrm{~dB}$, which is less than the simulated value. The measurements are aimed to produce shaped beams from an analog phased array without calibration. Calibrating the mmWave phased array in mass production is impractical due to the cost involved. The measurement shows that the designed phased array produces results close to the simulated plots, and calibration is not essential.

\section{CONCLUSION}

A simple computation procedure is proposed to generate user-specified single and multiple beams from a $5 \mathrm{G}$ mmWave planar array in 3D space. This method is capable of steering the multiple beams independently to any arbitrary angle in 


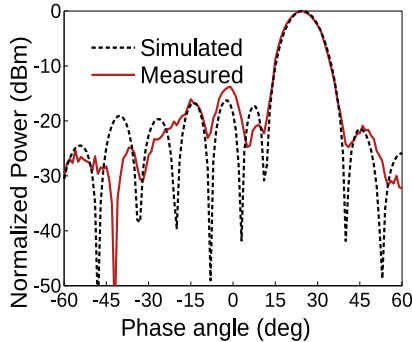

(a)

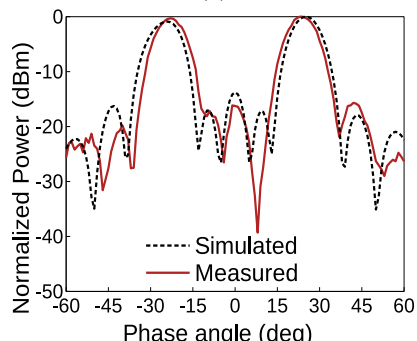

(c)

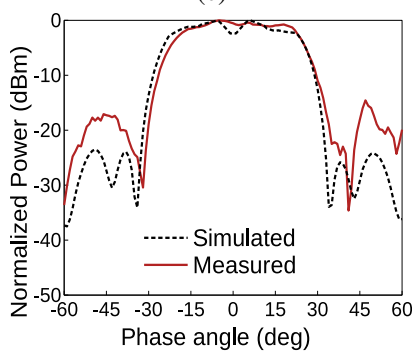

(e)

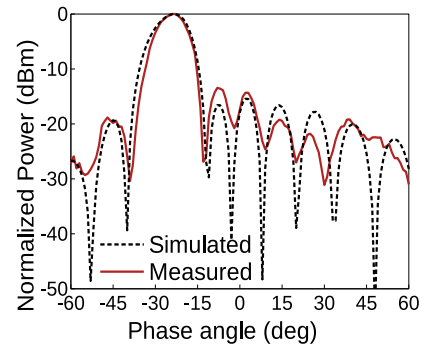

(b)

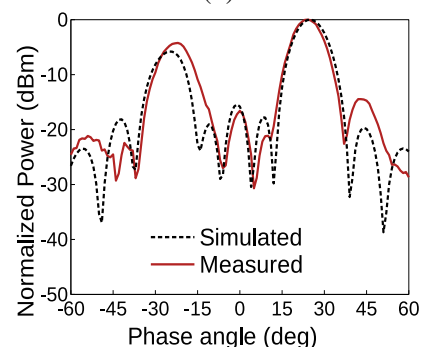

(d)

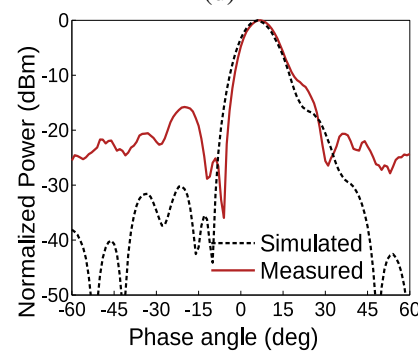

(f)
Fig. 12. Measured and simulated shaped beams at $28 \mathrm{GHz}$ on a $8 \times 8$ planar array with $0.65 \lambda$ element spacing. (a) Single beam $\left(+25^{\circ}\right)$. (b) Single beam $\left(-25^{\circ}\right)$. (c) Equal dual beams $\left(\theta= \pm 25^{\circ}\right)$. (d) Unequal beams $\left(\theta= \pm 25^{\circ}\right)$. (e) Sector beam. (f) Cosecant square pattern.

$\theta, \phi$ planes with equal and unequal power levels. Shaped beam patterns are realized using multiple beams approach. Shaped beam patterns are demonstrated by controlling the power, direction, and SLL of the independent beam peaks calculated from the proposed method. An evolutionary algorithm is proposed to find the number of optimum beams, peaks and their respective locations to synthesize the shaped beam pattern with a minimum ripple as low as $0.2 \mathrm{~dB}$. An $8 \mathrm{x} 8$ element planar array operating at $28 \mathrm{GHz}$ for mmWave $5 \mathrm{G}$ application is designed and used to demonstrate the suitability of the proposed method for, single, multiple, and shaped beam radiation patterns. Single, dual equal, dual unequal, sector, and $c s c^{2}$ patterns are generated and scanned from the developed mmWave phased array system. The proposed multibeam generation method is helpful for a base station to address multiple UEs simultaneously. The radiated power (EIRP) can be unequally shared between multiple beams to effectively address the near and far UE units to get maximum throughput. Multiple beams on the proposed $2 \times 2$ hybrid beamforming can serve multiple data strems and multiple users simultaneously with improved frequency diversity.

\section{REFERENCES}

[1] R. J. Mailloux, Phased array antenna handbook. Artech house, 2017.

[2] A. W. Rudge, "Multiple-beam antennas: Offset reflectors with offset feeds," IEEE Trans. Antennas Propag., vol. 23, pp. 317-322, May 1975.
[3] J. Ala-Laurinaho et al., "2-D Beam-Steerable Integrated Lens Antenna System for 5G E -Band Access and Backhaul," IEEE Trans. Microw. Theory Techn., vol. 64, no. 7, pp. 2244-2255, 2016.

[4] J. Blass, "Multidirectional antenna - A new approach to stacked beams," IRE Int. Conv. Rec., vol. 1, pp. 48-50, Mar. 1960.

[5] J. Butler, R. Lowe, "Beam-forming matrix simplifies design of electronically scanned antenna," Elect. Design, vol. 9, pp. 170-173, Apr. 1961.

[6] W. Rotman, R. F. Turner, "Wide-angle microwave lens for line source applications," IEEE Trans. Antennas Propagat., vol. 11, pp. 623-632, 1963.

[7] M. I. Skolnik, "Introduction to radar systems," New York, McGraw Hill Book Co.,, 1980.

[8] R. Elliott and G. Stern, "A new technique for shaped beam synthesis of equispaced arrays," IEEE Trans. Antennas Propagat., vol. 32, no. 10, pp. 1129-1133, 1984.

[9] W. Stutzman, "Synthesis of shaped-beam radiation patterns using the iterative sampling method," IEEE Trans. Antennas Propagat., vol. 19, no. 1 , pp. 36-41, 1971

[10] F. J. Ares-Pena, J. A. Rodriguez-Gonzalez, E. Villanueva-Lopez, and S. Rengarajan, "Genetic algorithms in the design and optimization of antenna array patterns," IEEE Trans. Antennas Propagat., vol. 47, no. 3, pp. 506-510, 1999.

[11] Y. Chen, S. Yang, and Z. Nie, "The application of a modified differential evolution strategy to some array pattern synthesis problems," IEEE Trans. Antennas Propagat., vol. 56, no. 7, pp. 1919-1927, 2008.

[12] P. Woodward, "A method of calculating the field over a plane aperture required to produce a given polar diagram," J. Inst. Electr. Eng. -Part IIIA: Radiolocation, vol. 93, no. 10, pp. 1554-1558, 1946.

[13] H. Orchard, R. Elliott, and G. Stern, "Optimising the synthesis of shaped beam antenna patterns," in IEE Proceedings H (Microwaves, Antennas and Propagation), vol. 132, pp. 63-68, IET, 1985.

[14] Y. T. Lo and S. Lee, Antenna Handbook: theory, applications, and design. Springer Science \& Business Media, 2013.

[15] J.-Y. Li, Y.-X. Qi, and S.-G. Zhou, "Shaped beam synthesis based on superposition principle and taylor method," IEEE Trans. Antennas Propagat., vol. 65, no. 11, pp. 6157-6160, 2017.

[16] W. Roh, J.Y. Seol, J. Park et al., "Millimeter-wave beamforming as an enabling technology for $5 \mathrm{G}$ cellular communications: Theoretical feasibility and prototype results," IEEE Commun. Mag., vol. 52, no. 2, pp. 106-113, 2014.

[17] K. Kibaroglu, M. Sayginer, T. Phelps, and G. M. Rebeiz, "A 64-element $28-\mathrm{GHz}$ phased-array transceiver with 52-dBm EIRP and 8-12-Gb/s 5G link at 300 meters without any calibration," IEEE Trans. Microw. Theory Techn., vol. 66, pp. 5796-5811, Dec. 2018.

[18] S. Prasad, M. Meenakshi, N. Adhithiya, Rao, P.H, R. Krishna Ganti, and S. Bhaumik, "mmWave multibeam phased array antenna for $5 \mathrm{G}$ applications," J. Electromagn. Waves Appl., pp. 1-13, 2021.

[19] X. Gu et al., "A multilayer organic package with 64 dual-polarized antennas for $28 \mathrm{GHz} 5 \mathrm{G}$ communication," Proc. IEEE IMS, pp. 18991901, Jun. 2017.

[20] B. Sadhu et al., "A 28-GHz 32-element TRX phased-array IC with concurrent dual-polarized operation and orthogonal phase and gain control for 5G communications," IEEE J. Solid-State Circuits, vol. 52, pp. 3373-3391, Dec. 2017.

[21] Ansys®, High Frequency Electromagnetic Simulation Software (HFSS), "Ansys (Release R1)," 2020.

[22] C. A. Balanis, Antenna Theory: Analysis and Design. John wiley \& sons, 2016.

[23] R. S. Elliot, “Antenna theory and design Prentice-Hall,” Inc., Englewood Cliffs, New Jersey, 1981.

[24] R. Storn and K. Price, "Differential evolution-a simple and efficient heuristic for global optimization over continuous spaces," J. Glob. Optim., vol. 11, no. 4, pp. 341-359, 1997. 